\title{
Stability of the Defect Renewal Volterra Integral Equations
}

\author{
R. S. Anderssen, F. R. de Hoog and M. Westcott \\ CSIRO Mathematics, Informatics and Statistics, GPO Box 664, Canberra, ACT 2601
}

Email: Frank.deHoog@csiro.au

\begin{abstract}
Ernest Filip Oskar Lundberg (1876-1965) is regarded as the founder of mathematical ruin theory (Cramer (1969)). In his 1903 thesis, and subsequently, Lundberg formulated and analysed probabilistic models for the dynamics of insurance practice and applied his ideas to the activities of insurance companies with which he was directly involved. As Cramer (1969) comments "his [Lundberg's] ideas were far ahead of his time, and his works deserve to be generally recognized as pioneering works of fundamental importance". His models represent early examples of stochastic processes with independent increments, before the development in the 1930's of a rigorous and general theory of stochastic processes by Kolmogorov, Khinchine, Levy and Feller. For example, the Poisson process, unknown as an explicit entity until its formal 1909 introduction by Erlang in the study of telephone traffic, comes out as a quite special case of the general probabilistic process formulated in the 1903 thesis (Cramer (1969)).

In insurance modelling, the goal is the prediction of the probability of various outcomes related to the dynamics and viability of a proposed operational scenario. An example of such an outcome is the probability of ultimate ruin. Mathematically, the underlying equations which must be solved take the form of renewal and defect renewal equations which form special subsets of linear convolution Volterra integral equations. The analysis of such equations has established that the required probability is often the tail of a related compound distribution. For the estimation of the probability of ultimate ruin, it is the tail of a compound geometric distribution which must be determined (e.g. section 7.3 in Willmot and Lin (2001)).

As discussed in considerable detail in Willmot and Lin (2001) and Psarrakos (2009, 2010), the renewal process that models the probability of ultimate ruin takes the form of a defect renewal equation. The defect parameter $\phi, 0<\phi<1$, characterizes the robustness of the insurance portfolio with $\phi \sim 1$ corresponding to a highly risky strategy.

Feller (1941) proposed and gave the first rigorous treatment of the renewal equation from a linear second kind convolution Volterra integral equation perspective, assuming the non-negativity of the kernel, forcing term and solution. His motivation was the 1939 papers of Lotka, which comprehensively summarized the early history of the renewal equation and motivated, via a number of different applications, the importance of the Volterra structure of such equations. For the types of stochastic processes examined by Feller, the defect renewal structure is pathological as it corresponds to some of the components having a zero probability of failure.

In insurance as well as other stochastic process applications, it is often the case that only an estimate of the kernel of the (defect) renewal equation will be available from experimental data. This leads naturally to the need to examine the stability of the solution of the corresponding defect equations with respect to perturbations in their kernels, which is the focus of this paper. The goal is to show how the earlier analyis of Feller (1941) of the Volterra structure of the renewal equations extends naturally to the analysis of the defect renewal equation. This is achieved using the resolvent kernel relationship for linear second kind convolution Volterra integral equations, specialized to the situation where the kernel, forcing term and solution are non-negative. It builds on and complements the stability analysis of the interconversion equation of rheology and of its extension to linear first and second kind convolution Volterra integral equations (Anderssen et al. (2008a,b)). It illustrates the strong overlap between the theories of stochastic processes and Volterra integral equations.
\end{abstract}

Keywords: second kind, convolution, Volterra, integral equations, renewal, defect renewal, kernel stability 


\section{THE DEFECT RENEWAL EQUATION}

Since the 1903 thesis of Lundberg, the dynamics of an insurance portfolio has been modelled in terms of the stochastic dynamics of the progressive sum

$$
S_{N_{t}}=X_{1}+X_{2}+X_{3}+\cdots X_{N_{t}}, \quad N_{t}=1,2, \cdots,
$$

of a sequence $X_{i}, i=1,2, \cdots$ of independent and identically distributed (i.i.d.) random variables supported on $[0, \infty)$ which model the stochasticity of the incoming claims. The claims are assumed to arrive at random times $\left\{t_{i}\right\}, i=1,2, \cdots$, with inter-arrival times $\tau_{i}=t_{i}-t_{i-1}$ with $t_{0}=0$. If the $\left\{\tau_{i}\right\}$ are assumed to be i.i.d, then the arrivals of claims, by definition, form a renewal process. Usually, this process is assumed to be Poisson. The integer-valued random variable $N_{t}$ is the number of claims made in the time interval $(0, t]$. The viability of an insurance portfolio depends on whether or not the insurer's surplus

$$
U(t)=S^{*}+c t-S_{N_{t}}>0, \quad t>0,
$$

where $S^{*} \geq 0$ is the initial surplus and $c$ the premium rate per unit time. Ruin occurs if $U(t)$ ever becomes negative. Formally, for the random event

$$
\mathcal{M}=\min _{t \geq 0} U(t)
$$

the event $\mathcal{R}$ uin occurs if

$$
\mathcal{R u i n} \equiv\{\mathcal{M} \leq 0\} .
$$

Intuitively, $\mathcal{R}$ uin is certain if, on average, the payouts $S_{N_{t}}$ exceed income; namely, if $(\mathrm{E}[X]>c \mathrm{E}[\tau])$. If, however, $(\mathrm{E}[X]<c \mathrm{E}[\tau])$, there is a positive probability that $\mathcal{R}$ uin will not occur. The mean "drift" of the process $U(t)$ is defined by $(c \mathrm{E}[\tau]-\mathrm{E}[X])$, so negative drift corresponds to certain $\mathcal{R}$ uin and positive drift to a positive probability of survival.

Under these assumptions, the successive steps in the values of the surplus $\left(U\left(t_{i+1}\right)-U\left(t_{i}\right)\right)$ are clearly i.i.d. random variables, so the $U\left(t_{i}\right), i=1,2, \ldots$ correspond to a one-dimensional random walk starting from $S^{*}$. Embedded within this random walk is the random walk $Z_{i}^{\#}$ of the successively decreasing minima of the process $U(t)$, defined by $Z_{0}^{\#}=S^{*}$ and, for $k=1,2, \cdots$,

$$
Z_{k}^{\#}=\left\{\text { first value of surplus for which } U\left(t_{i}\right)<Z_{k-1}^{\#}\right\}
$$

If $U_{N_{t}}$ has a positive drift, there will be a last minimum with probability 1 . Indeed, the process might never drop below the initial value.

The succession of decreasing ladder steps (record increments) represent the losses defined between the minima. Define $\Delta_{k}^{\#}=Z_{k-1}^{\#}-Z_{k}^{\#}, k=1,2, \cdots$. They are non-negative and represent the magnitudes of the losses. As shown in Feller (cf. Feller (1971), Section 12.1), the $\Delta_{k}^{\#}$ form a sequence of nonnegative i.i.d. random variables, so the process

$$
Y_{n}^{\#}=\sum_{k=1}^{n} \Delta_{k}^{\#}, \quad n=1,2, \cdots,
$$

is by definition a renewal process. If there is a positive drift, the process is terminating (or transient); i.e. there is a positive probability $1-\phi$ that any ladder step is the final one and the renewal process has a finite lifetime $\mathcal{L}$. In this case, the common distribution of the $\Delta_{k}^{\#}$ is defective; i.e. it has an atom of probability $1-\phi$ at $+\infty$. Further, the distribution of $\mathcal{L}$ is compound geometric (Feller (1971), Section 11.6); i.e. it is the sum of a geometrically distributed number of i.i.d. random variables where the geometric distribution has parameter $\phi$. The common distribution of the summands, $F(x)$ say, for $x>0$, is the distribution of the non-defective part of the $\Delta_{k}^{\#}$; i.e.

$$
\phi F(x)=\operatorname{Pr}\left(\Delta_{k}^{\#} \leq x\right), \quad \text { for } \quad x>0 .
$$


Then the distribution of $\mathcal{L}, G(x)$ say, is

$$
G(x)=\operatorname{Pr}(\mathcal{L} \leq x)=(1-\phi) \sum_{n=0}^{\infty} \phi^{n} F^{n *}(x), \quad x<0,
$$

where $F^{n *}=\operatorname{Pr}\left(Y_{n}^{\#} \leq x\right)$ denotes the $n$-fold convolution of $F$ with itself.

To calculate the probability of ruin for an initial capital $x>0$ which is $\psi(x)=\operatorname{Pr}(\mathcal{L}>x)$, it is the tail distributon

$$
\bar{G}(x)=1-G(x)=(1-\phi) \sum_{n=0}^{\infty} \phi^{n} \overline{F^{n *}}(x), \quad \overline{F^{n *}}(x)=1-F^{n *}(x)
$$

that is required. It satisfies the defect renewal equation

$\bar{G}(x)=\phi \int_{0}^{x} \bar{G}(x-\tau) d F(\tau)+\phi \bar{F}(x), \quad \bar{F}(x)=1-F(x)$.

Assuming that $F \in C^{1}[0, \infty)$ with $F^{\prime}(x)=d F / d x$, this equation can be rewritten as

$$
\bar{G}(x)=\int_{0}^{x} k(x-s) \bar{G}(s) d s+\phi \bar{F}(x), \quad k(x)=\phi F^{\prime}(x) .
$$

It represents a specific realization of the second kind convolution Volterra integral equation

$y=k * y+f, \quad k * y=(k * y)(x)=\int_{0}^{x} k(x-s) y(s) d s=\int_{0}^{x} y(x-s) k(s) d s$,

with the kernel $k$, the solution $y$ and the forcing term $f$ non-negative functions, and $\|k\|_{1}=\phi<1$.

In addition to ruin analysis, the above defect renewal equation arises independently in a number of practical stochastic modelling situations including in reliability, branching and queuing. In an equilibrium queue, the compound geometric distribution models the distribution of the waiting times of arrivals. The essential stochastic dynamics is the same as that outlined above for an insurance portfolio.

The sequel has been organized in the following manner. The effect on the solution of equation (3) of perturbations in the kernel $k$ is examined in section 2. An interpretation of the resulting stability results, as they relate to the defect renewal equation (2), is given in section 3.

\section{SOlution STABILITY With RESPECT TO KERNEL PERTURBATIONS}

Differentiation with respect to the independent variable $x$ will be denoted by $k^{\prime}=d k(x) / d x, k^{\prime \prime}=$ $d^{2} k(x) / d x^{2}$, etc. It will be assumed that the relevant functions have the required smoothness.

In his analysis of the renewal equation, Feller (1941) exploited the properties of second kind convolution Volterra integral equations with kernel, solution and forcing terms being non-negative, and, thereby, established the existence and uniqueness of and various asymptotic properties for the solution for such equations. However, Feller did not explicitly examine the defect form of the renewal equation or consider the effect of perturbations in the kernel $k$ on the solution $y$. From a practical perspective, Feller's focus was Lotka's method for solving renewal equations.

There are various ways in which the effect of kernel perturbation can be estimated. In de Hoog and Anderssen (2011), bounds have been derived directly for the second kind equation (3), under the assumption that the kernel $k$ and the forcing term $f$ satisfy $k \geq 0$ and $f \geq 0$, and $\|k\|_{1}=\phi$. Here, it is shown how bounds can be derived using the first kind convolution Volterra integral equation

$K * y=F, \quad F(0)=0, \quad K=K(x), \quad K(0)=1, \quad y=y(t)$.

Differentiation of this last equation yields

$$
y+K^{\prime} * y=F^{\prime}
$$


which, with

$K(x)=1-\int_{0}^{t} k(\bar{x}) d \bar{x}, \quad F(t)=\int_{0}^{t} f(\bar{x}) d \bar{x}$,

yields equation (3). Since it has been assumed above that $k \geq 0$ and $f \geq 0$, it follows that

$$
K(x) \geq 0 \text { if }\left(1-\int_{0}^{x} k(\bar{x}) d \bar{x}\right) \geq 0 ; \quad F \geq 0 ; \quad F^{\prime} \geq 0 .
$$

With $\|k\|_{1}=\phi$, the conditions

$K(t) \geq 0, \quad K^{\prime}(t) \leq 0, \quad K \in C^{1}[0, \infty) \quad$ and $\quad K(\infty)=1-\phi$

are automatically satisfied when the kernel $k$ is a defect density function.

The framework developed by de Hoog and Anderssen (2010) and Anderssen et al. (2011) for the analysis of kernel perturbations for first kind Volterra convolution integral equations can now be utilized. The key step is the assumption that, for the given kernel $K$, there exists a function $H$ with respect to which the following interconversion relationship holds

$$
H * K=K * H=t .
$$

As an immediate consequence, since $H * K * y=t * y=H * F$, it follows that de Hoog and Anderssen (2010)

$$
y=\frac{d^{2}}{d t^{2}}(H * F) \text {. }
$$

Corresponding to the first kind equation (4), the effect of kernel perturbations on its solution is assumed to take the form

$(K+\gamma) *(y+\epsilon)=F, \quad \gamma(x)=-\int_{0}^{x} \delta k(\bar{x}) d \bar{x}, \quad \gamma(0)=0$,

from which it follows, again using $H * K=t$, that

$K * \epsilon=-y * \gamma-\epsilon * \gamma$,

and, hence,

$\epsilon=-\frac{d^{2}}{d t^{2}}(H * y * \gamma+H * \epsilon * \gamma)$.

On the basis of the analysis found in de Hoog and Anderssen (2010), it follows, for $\zeta<1$, that, because of the validity of the conditions (6),

$$
\begin{aligned}
\|\epsilon\|_{\infty} & \leq \frac{\beta}{1-\zeta}\|\gamma\|_{\infty}, \quad\|\gamma\|_{\infty}=\max _{0 \leq x \leq \infty}\left|\int_{0}^{x} \delta k(\bar{x}) d \bar{x}\right|, \quad\|\gamma\|_{1}=\int_{0}^{\infty}\left|\int_{0}^{x} \delta k(\bar{x}) d \bar{x}\right| d x, \\
\text { and } & \\
\|\epsilon\|_{\infty} & \leq \frac{\zeta}{1-\zeta}\|y\|_{\infty},
\end{aligned}
$$

with, using the above properties of $\gamma$,

$$
\beta=\frac{|y(0)|+\left\|y^{\prime}\right\|_{1}}{1-\phi}, \quad \zeta=\frac{\|\delta k\|_{1}}{1-\phi}, \quad \frac{\zeta}{1-\zeta}=\frac{\|\delta k\|_{1}}{1-\phi-\|\delta k\|_{1}} .
$$

From the first result (10), it is clear that, when $\delta k$ is oscillatory with changing sign, the size of $\|\gamma\|_{\infty}$, and hence, $\|\epsilon\|_{\infty}$ (especially when $\phi<<1$ ), can be quite small. The second result (11) has a similar form to the corresponding result in de Hoog and Anderssen (2011), which was derived using the basic properties of second kind convolution Volterra integral equations (3) for which the kernel $k$, the solution $y$ and the forcing term $f$ are non-negative functions, and $\|k\|_{1}=\phi<1$. 


\section{Conclusions}

The clear advantage of the bounds (10) and (11) is that they highlight, respectively, the dependence of $\|\epsilon\|_{\infty}$ on $\|\gamma\|_{\infty}$ and on $\|y\|_{\infty}$. In addition, they show that the dependence of $\|\epsilon\|_{\infty}$ on the form of the kernel $k$ is through the value of $\phi$.

Because of the explicit dependence of $\|\epsilon\|_{\infty}$ on the reciprocal of $(1-\phi)$, it follows that as an insurance protfolio becomes more problematic as $\phi \rightarrow 1$, the use of approximations for $k=\phi F^{\prime}$, even with very small errors, will, in general, yield unrelaible estimates for the probability of total ruin. This is a direct reflection of the volatility of situations where $\phi \sim 1$.

Here, the above analysis is based on the assumption that $\phi$ is fixed. In a more general treatment, the effect of how perturbations in the assumed claim size distributions affect the possible values of $\phi$ would have to be taken into account.

\section{REFERENCES}

Anderssen, R. S., A. R. Davies, and F. R. de Hoog (2008a). On the sensitivity of interconversion between relaxation and creep. Rheologica Acta 47, 159-167.

Anderssen, R. S., A. R. Davies, and F. R. de Hoog (2008b). On the Volterra integral equation relating creep and relaxation. Inverse Problems 24, 035009 (p. 13).

Anderssen, R. S., A. R. Davies, and F. R. de Hoog (2011). The effect of kernel perturbations when solving the interconversion convolution equation of linear viscoelasticity. Appl. Math. Lett. 24, 71-75.

Cramer, H. (1969). Historical review of Filip Lundberg's work on risk theory. Skandinavisk Akuarietidskrift No. 3-4 Suppl., 6-12.

de Hoog, F. R. and R. S. Anderssen (2010). Kernel perturbations for Volterra convolution integral equations. J. Int. Eqns. Appl. 22, 427-441.

de Hoog, F. R. and R. S. Anderssen (2011). Kernel perturbations for Volterra convolution integral equations. Preprint.

Feller, W. (1941). On the integral equation of renewal theory. Annals Math. Stats. 12, 243-267.

Feller, W. (1971). An Introduction to Probability Theory and its Applications, Volume II. John Wiley \& Sons, New York.

Psarrakos, G. (2009). Asymptotic results for heavy-tailed distributions using defective renewal equations. Stats. \& Prob. Let. 79, 774-779.

Psarrakos, G. (2010). On the DFR property of the compound geometric distribution with applications in risk theory. Insurance Math. \& Econ. 47, 428-433.

Willmot, G. E. and X. S. Lin (2001). Lundberg Approximations for Compound Distributions with Insurance Applications. Springer, New York. 